\title{
PIC simulations of plasma interaction with shaped and unshaped gaps in TEXTOR
}

\author{
M. Komm ${ }^{1,2}$, R. Dejarnac ${ }^{2}$, J.P. Gunn ${ }^{3}$, A. Kirschner ${ }^{4}$, A. Litnovsky ${ }^{4}$, D. \\ Matveev $^{5}$ and Z. Pekarek ${ }^{1}$ \\ ${ }^{1}$ MFF Charles University, V Holešovičkách 2, 18000 Prague 8, Czech Republic \\ ${ }^{2}$ Institute of Plasma Physics AS CR, v.v.i., Za Slovankou 3, 18200 Prague 8, Czech Republic \\ ${ }^{3}$ CEA, IRFM, F-13108 Saint-Paul-lez-Durance, France \\ ${ }^{4}$ Institut für Energieforschung - Plasmaphysik, Forschungszentrum Jülich, Trilateral Euregio \\ Cluster, Association EURATOM-FZ Jülich, D-52425 Jülich, Germany \\ ${ }^{5}$ Department of Applied Physics, Ghent University, Plateaustraat 22, B-9000 Ghent, Belgium \\ E-mail: kommeipp.cas.cz
}

\begin{abstract}
This paper presents particle-in-cell simulations of the plasma behaviour in the vicinity of gaps in castellated plasma-facing components (PFCs). The point of interest was the test limiter of the TEXTOR tokamak, a PFC designed for studies of plasma-wall interactions, in particular, related to impurity transport and fuel retention. Simulations were performed for various plasma conditions in the vicinity of the castellated surface, where the gaps can be either shaped or unshaped. It was observed that depending on plasma parameters the transport of plasma particles inside the gap can be either in potential- or geometry-dominated regimes. The mechanisms responsible for the formation of a potential peak inside the poloidal gap and its consequences on plasma deposition profiles are discussed. Study of gap shaping was performed in order to validate its effectiveness.
\end{abstract}

Submitted to: Plasma Phys. Control. Fusion 


\section{Introduction}

Fuel retention in nuclear fusion devices is a subject of intensive research, especially because of implications for ITER and future reactors [1], which will operate with tritium. For safety reasons, the amount of tritium inside the ITER vessel is restricted to $700 \mathrm{~g}$, and its accumulation leads to a limitation of the number of discharges which can be performed before the safety limit is reached. Current experiments show that the fraction of retained fuel varies between 2 - 20 percent [1] depending on the machine and method used for analysis. The plasma facing components (PFCs) have to be castellated - i.e. split into small blocks separated by gaps (see Fig. 1), in order to withstand thermal stresses caused by plasma heat loads and induced currents. Measurements at DIII-D [2] show that up to $40 \%$ of the retained fuel can be trapped in gaps. Each wall material has its own mechanism of fuel retention, the highest rate was observed for carbon, which can form hydrocarbon layers on shadowed surfaces. Such layers can grow one on another and as such there is no saturation. Moreover, gaps are difficult to access by cleaning methods, so minimizing the deposition there is of high interest. Recent studies of the deuterium retention inside the gaps were performed at the TEXTOR tokamak with the so-called test limiter [3] [4]. The test-limiter has regular rectangular gaps as well as shaped gaps, where the leading edge of the gap is hidden from direct plasma flux. Such shaping is expected to reduce in-gap deposition (see Fig. 1).

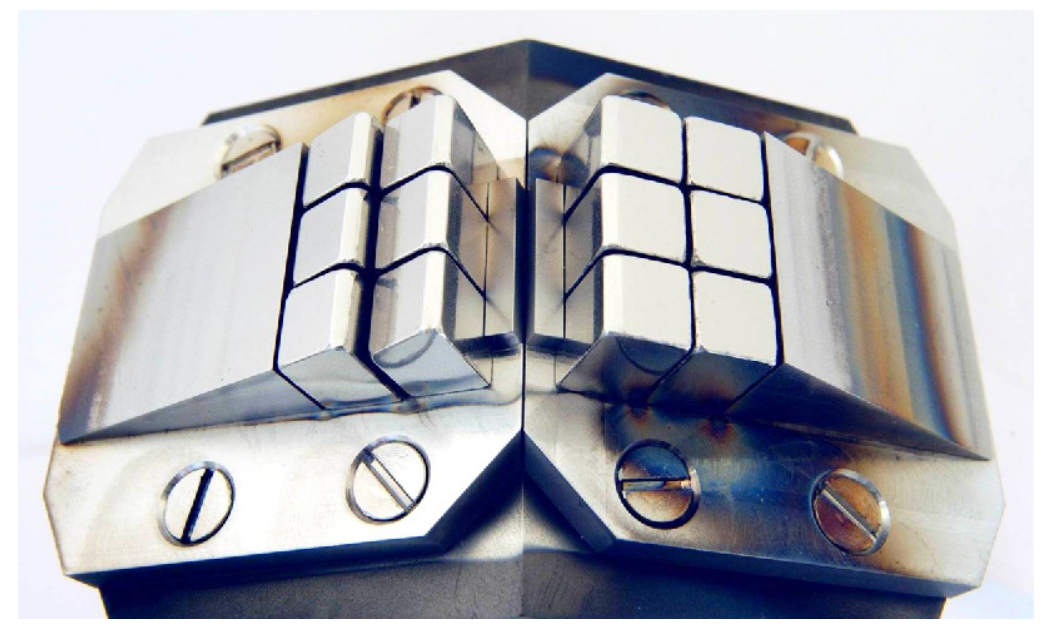

Figure 1. The TEXTOR test limiter with shaped gaps on the left and unshaped gaps on the right side. Toroidal magnetic field is oriented in the horizontal direction.

The shaping used in this experiment is more pronounced than could be of use in ITER (the top surface of each tile has an inclination of 40 degrees with respect to the magnetic field, in contrast to 2-4 degrees in the ITER divertor). The reason for such exaggerated shaping was to demonstrate an influence on deposition profiles. The limiter tiles were exposed to plasma and then analysed using post-mortem techniques such as the secondary ion mass spectrometry (SIMS), nuclear reaction analysis (NRA) and electron probe microanalysis (EPMA). The amount of deposited hydrocarbon layers and their fuel content were measured. In order to 
explain the deposition mechanisms, a 3D Monte-Carlo neutral impurity transport code 3DGAPS, was developed [5].

Modeling of transport inside the gaps involves a number of parameters which are often not known from experiment. In order to make the simulations realistic, the distribution of particle fluxes coming from the main plasma is needed. The aim of this work is to study plasma behaviour in the vicinity of poloidal gaps for the range of plasma conditions typically met in the TEXTOR tokamak scrape-off layer (SOL) by using the SPICE2 code, which implements the particle-in-cell method of numerical simulations [6]. The results of the simulations have been used in [5] to provide realistic profiles of plasma fluxes inside the gaps.

Fuel retention due to hydrocarbon layer formation makes carbon a problematic material for the tritium phase of ITER and next-step devices. The ITER divertor will be most probably made of tungsten, a high-Z impurity, which can cause the plasma to disrupt even at small concentrations. The aim of the experiments related to simulations presented in this paper is to find tile geometry with reduced retention inside the gaps, which would enable use of carbon with DT plasmas.

\section{SPICE2 setup}

The SPICE2 code [7] is a 2D cartesian Particle-In-Cell code developed in collaboration between the Institute of Plasma Physics AS CR in Prague and CEA Cadarache, France. It is designed to simulate sheath and magnetic pre-sheath of PFCs in tokamaks. Such PFCs usually have surfaces at oblique angles with respect to the magnetic field lines, which requires special care during particle injection to ensure that the gyro-phase of particles is distributed uniformly. Ions are injected into the simulation region with a distribution of velocities parallel to the magnetic field given by a 1D quasineutral kinetic model of the SOL that satisfies Bohm's criterion [8]. The perpendicular component of the ion velocities and all three components of the electron velocities are Maxwellian. SPICE2 has been previously benchmarked with experiment [9] and used to study impact of edge-localized modes on ITER PFCs [10]. There are no collisions included in the simulations, the mean free path of electrons for the studied plasma parameters is much larger than the gap width and as such does not influence the plasma deposition inside the gaps.

The general geometry of SPICE2 gap simulations consists of an injection plane (top boundary in Fig. 2) with imposed value of plasma potential $V_{p l}=0 k T_{e}$, two periodic side boundaries and a bottom absorbing equipotential wall at floating potential $V_{f l}=-3 k T_{e}$. While the real value of floating potential may vary depending on the coefficient of secondary electron emission, test simulations have shown little sensitivity of the plasma deposition profiles on the exact value of the floating potential within the range from -2.5 to $-3.5 k T_{e}$. In the simulated region, there are two electrodes representing the tiles. Experiments with the TEXTOR test limiter have been done with unshaped (Fig. 2A) and shaped (Fig. 2B) gaps to investigate the impact of shaping on the plasma deposition inside the gaps. The gaps used in the experiments are $0.5 \mathrm{~mm}$ wide and the tiles are $10 \mathrm{~mm}$ long. The magnetic field is inclined at 20 degrees with respect to the flat top surface of the unshaped gap. In the case of 
shaped gaps, the top surfaces are inclined such that one is parallel to the magnetic field and the other one has an inclination of 40 degrees with respect to the field lines. In the described experiment, all the gaps were more than $10 \mathrm{~mm}$ deep, however only the first $2 \mathrm{~mm}$ were simulated, as the plasma does not penetrate any deeper.
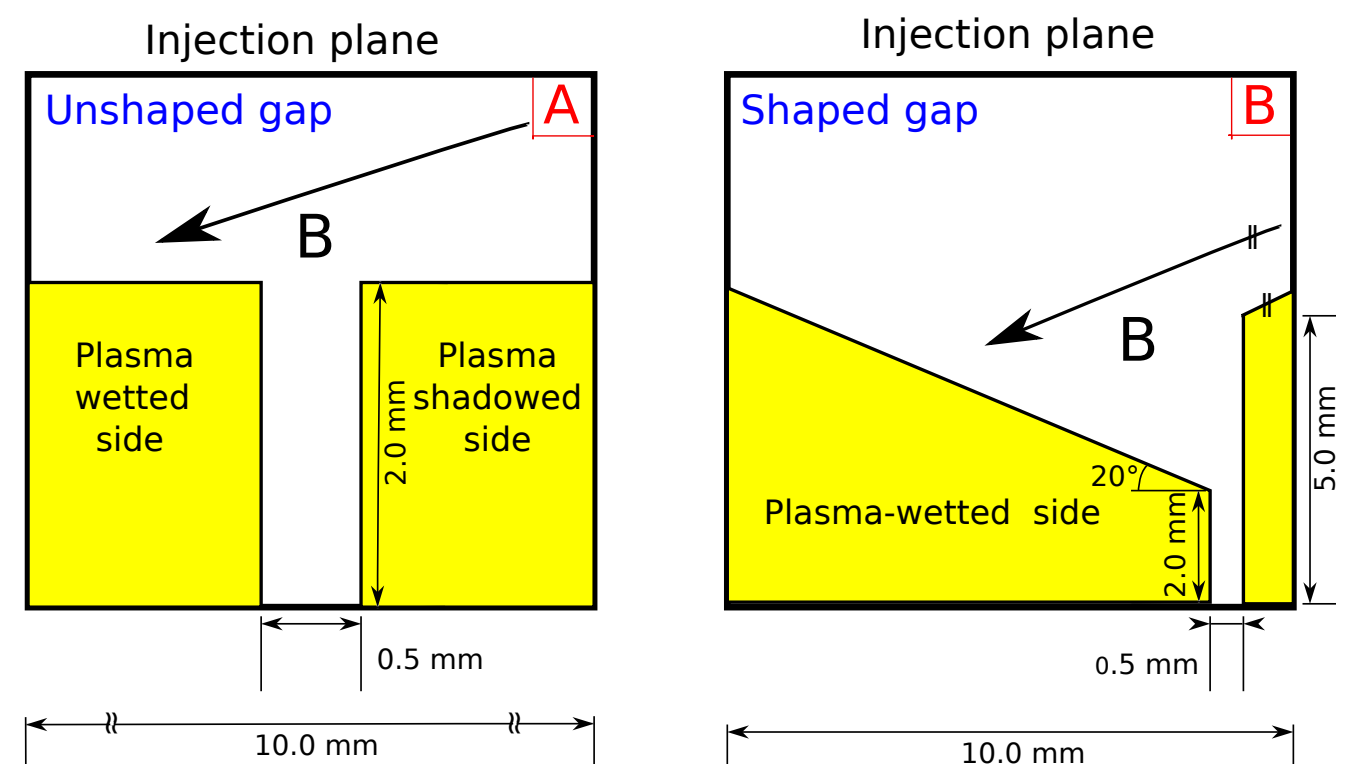

Figure 2. Geometry of the gaps. A - unshaped gaps, B - shaping used in TEXTOR test limiter.

\section{Results of simulations}

\subsection{Unshaped gaps}

3.1.1. Standard case First, let us study the effect of this gap geometry on the plasma deposition profiles for fixed plasma conditions. We define the standard case with typical conditions of the TEXTOR SOL as follows:

- Plasma density $n=6 \times 10^{18} \mathrm{~m}^{-3}$

- Electron temperature $T_{e}=30 \mathrm{eV}$

- Deuterium ion temperature $T_{i}=60 \mathrm{eV}$

- Magnetic field magnitude $B=2.25 \mathrm{~T}$

- Magnetic field inclination $\alpha=20$ degrees with respect to the top surface of an unshaped gap

The main mechanism governing plasma behaviour in the vicinity of the gaps is the gyromotion of particles. Ions have Larmor radii comparable to the size of the gap $\left(r_{L_{i}}=0.35 \mathrm{~mm}\right)$ enabling them to penetrate into the shadowed region. Electrons are strongly magnetized and simply follow the field lines, as there is no mechanism of cross-field transport implemented in the model. This Larmor effect results in net positive space charge inside the gap and subsequent formation of a potential peak (as seen in Fig. 3). Incoming deuterium ions can be 
divided into three categories - some hit the plasma wetted side of the gap $\left(\Gamma_{a}\right)$, others fall onto the shadowed gap side $\left(\Gamma_{b}\right)$ and the rest is deflected from the gap and hit the top surface of the tiles $\left(\Gamma_{c}\right)$ [7]. We can calculate the total expected flux inside the gap by taking a fraction of all injected particles, which corresponds to the ratio of the gap size to the simulation width in the $y$ direction

$$
\Gamma_{t o t}=\Gamma_{a}+\Gamma_{b}+\Gamma_{c}=\Gamma_{i n j} \frac{L_{g a p}}{L_{y}} .
$$

The profiles of $D^{+}$fluxes along the gap sides are shown on Fig. 4 (black lines). The left side of the gap, which is open to direct plasma flux $\Gamma_{a}$, shows a complex decay structure. Near the entrance, there is a plateau region followed by an exponential decay. The transition occurs at the projection of the lowest magnetic field line which is not intercepted by the right tile. The plateau is formed due to ions which are prevented from flowing deeper in the gap by the potential peak. We also observe an ion flux $\Gamma_{b}$ falling on the right side of the gap, although it is in the plasma shadow. The total amount of flux is approximately one order of magnitude lower than that on the plasma wetted side. The distribution of particle fluxes shows that typically $5 \%$ of particles reach the right side of the gap, $75 \%$ the left side of the gap and the remaining $20 \%$ are prevented from entering the gap and hit the top surface.

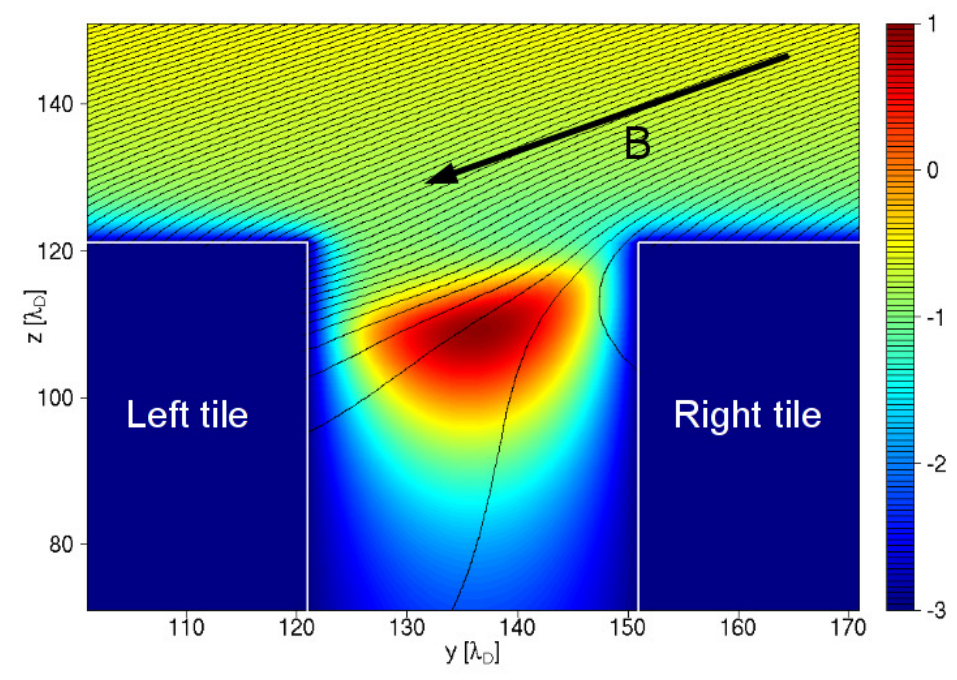

Figure 3. Potential profile of the standard case. Black lines represent deuterium ion streamlines. Potential in $k T_{e}$, dimensions in Debye lengths. In absolute units the gap is 0.5 mm wide.

In order to explain the mechanism which can lead to particle flux $\Gamma_{b}$ falling onto the shadowed side of the gap, we have followed the magnetic field line which passes close to the corner of the right tile (see Fig. 5 left). The total electric field felt by an imaginary sensor which would follow this field line, divides the trajectory into three sections as we can see in Fig. 5 (right), plotted as a function of parallel coordinate $l$. Far from the gap $(l>150)$ the total electric field is positive and first acts towards the tiles (there is a regular sheath potential drop). Once the sensor enters the gap $(125<l<150)$, the electric field changes sign and 

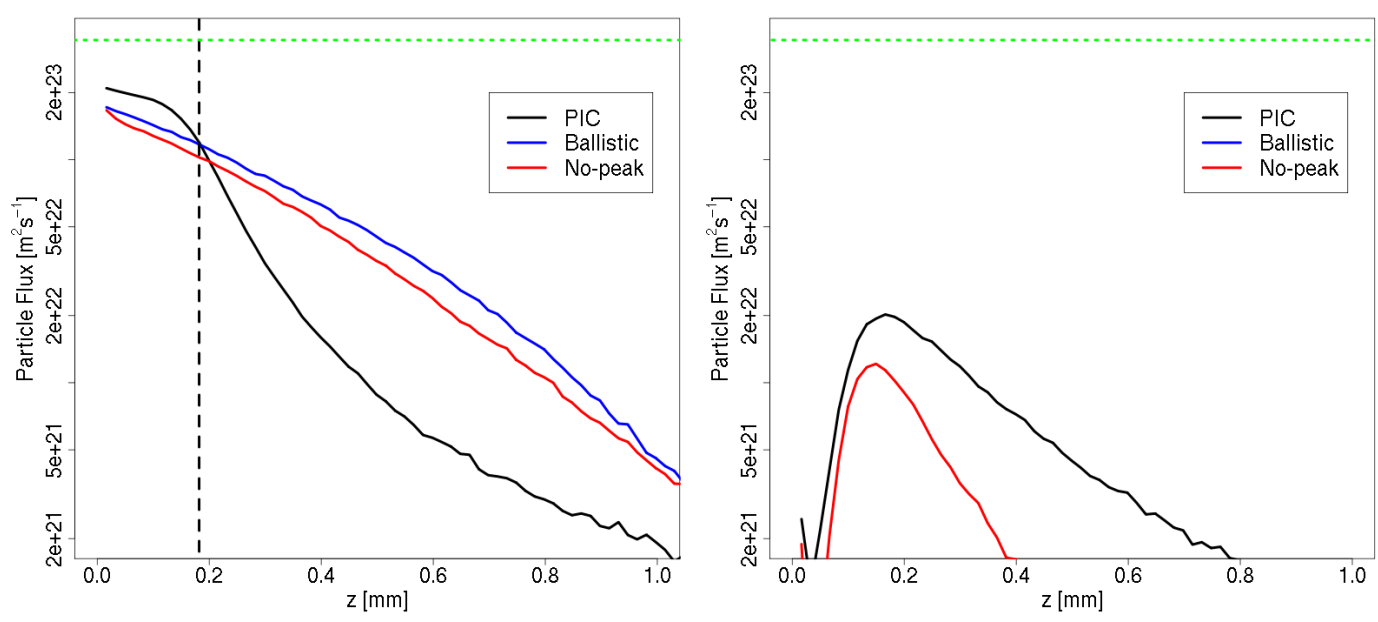

Figure 4. Deuterium fluxes along the gap on the plasma wetted side (left) and plasma shadowed side (right). Black curves represent the regular PIC simulation, red curves simulation without the potential peak and blue curves the ballistic case. Gap entrance corresponds to $z=0 \mathrm{~m}$. Black dashed line shows the geometrical projection of plasma wetted area, the green dotted line shows projection of incoming particle flux on the gap side.

points in the direction of the plasma shadowed side. Finally, when the sensor approaches the plasma wetted side of gap $(l<125)$, it changes sign again and acts towards the surface. The arrows representing the direction and magnitude of the local electrical field show that there is indeed a region inside the gap, where the field attracts ions towards the plasma shadowed side of the gap. Since the ion Larmor radius is comparable to the gap size and so much larger than the typical scale of variation of the local strong electric field, the ions behave as demagnetized inside the gap.
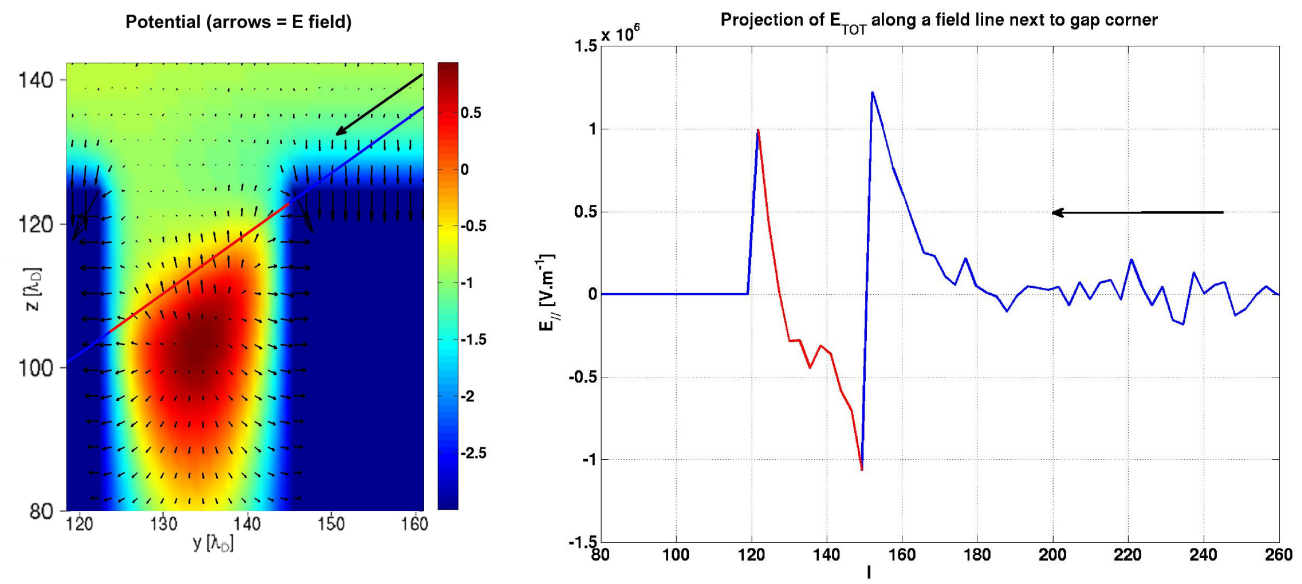

Figure 5. Detail of electrical potential in the vicinity of the gap (left) with local electric field (arrows) and the projection of the total electric field on a B field line passing next to the tile corner (right). 
In order to investigate the influence of the potential peak and sheath electric fields on the plasma penetration into the gap, the PIC simulation has been compared with two special nonselfconsistent cases with fixed imposed potential. One is the ballistic case (with zero electric field), second is a case with potential similar to the steady-state result of the PIC simulation but the potential peak has been manually erased. The latter will be called no-peak further in the text. Fig. 4 shows comparison of the three cases. It is visible that ions penetrate deeper in the gap in the no-peak case compared to the self-consistent PIC simulation. This demonstrates that the potential peak acts as a barrier that regulates the ion flux according to its magnitude. It is also partially responsible for the ion flux, which is pushed towards the plasma shadowed side. In both cases, the total fraction of ions deposited inside the gap is $\sim 80 \%$, which means that the fraction of deflected particle remains almost unchanged $(\sim 20 \%)$.

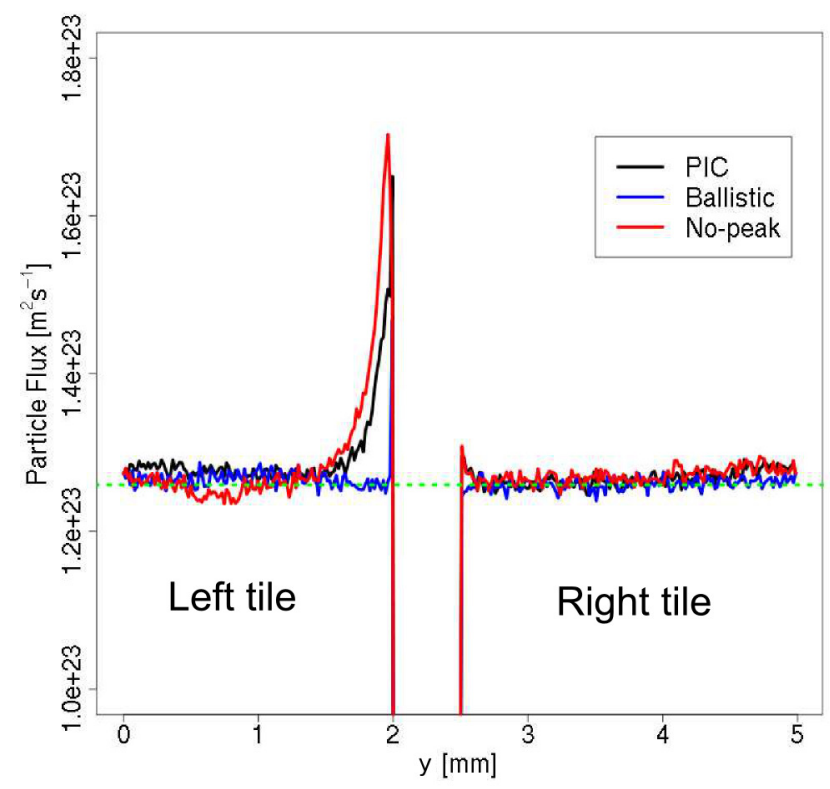

Figure 6. Deuterium fluxes falling onto the top tile surface. Black curves represent the regular PIC simulation, red curves simulation without the potential peak and blue curves the ballistic case. Green dotted line shows the projection of incoming particle flux onto the top surface.

In the ballistic case, there are no particles hitting the plasma shadowed side and the particle flux on the plasma wetted side is increased compared to the PIC run. The fraction of deflected particles is bellow 5\%, which is the precision level of the diagnostics for flux recording. It can be seen that the fluxes in all three cases are lower than the unperturbed flux coming from plasma and falling onto a surface with the same orientation as the gap (dotted green line).

Particles $\Gamma_{c}$, which are deflected from the gap, reach the top surface of the next tile, where a peak of particle flux is observed in the vicinity of the tile corner (see Fig. 6). The corner peak is present for PIC and no-peak case, which indicates that is not caused by the presence of potential barrier at gap entrance. It is not present in the ballistic case, which confirms that 
the particles are deflected by the electric fields associated to sheath potential drop at the top tile surfaces.

These results lead to a conclusion that while the electric fields associated with the sheath in this complex geometry affect the fraction of particles which are prevented from entering the gap, the potential peak is responsible for the distribution of ion fluxes inside the gap. The combination of both mechanisms is responsible for the flux falling on the plasma shadowed side.

3.1.2. Parameter study A set of simulations with varying plasma parameters was performed in order to investigate the effect of different plasma conditions on the deposition profiles for the fixed geometry. The plasma density was varied over the range $2 \times 10^{18}$ and $1 \times 10^{19} \mathrm{~m}^{-3}$ and the electron temperature between 20 and $50 \mathrm{eV}$, which are characteristic for TEXTOR SOL. The magnitude and inclination of the magnetic field and ratio of temperatures was kept constant, same as in the standard case $(\tau=2)$. As shown in the standard case, the crucial element governing the particle fluxes along the gap is the potential peak near the entrance of the gap. The maximum value of the electric potential in the positive space charge region for all the studied cases is summarized in Fig. 7. A white dashed line, where the peak height is equal to the plasma potential, separates zones with different influence of the potential peak as it will be explained further in this section.

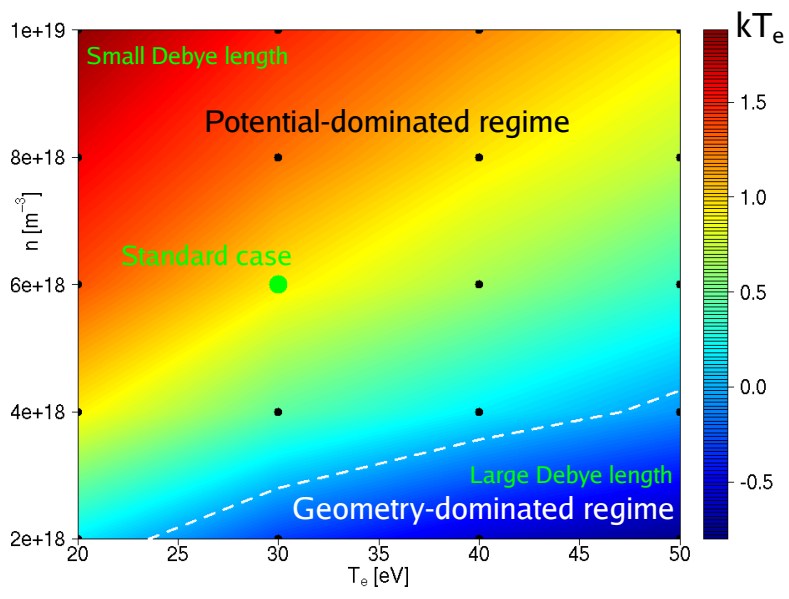

Figure 7. Maximum of potential at the potential peak inside the gap. Contour lines separate the zones of strong and weak influence of the peak.

The simulations reveal that the magnitude depends on the Debye length and for gap width comparable to the ion Larmor radius can be fitted by the following function:

$$
\phi_{\max }=A_{1} e^{A_{2} \sqrt{\lambda_{D}}},
$$

where $A_{1}$ and $A_{2}$ are fitting constants. To demonstrate the differences in plasma behaviour, we have selected two extreme cases - small Debye length $\lambda_{D}=1.05 \times 10^{-5} \mathrm{~m}\left(\mathrm{n}=1 \times 10^{19}\right.$ $\left.\mathrm{m}^{-3}, T_{e}=20 \mathrm{eV}\right)$ and large Debye length $\lambda_{D}=3.71 \times 10^{-5} \mathrm{~m}\left(\mathrm{n}=2 \times 10^{18} \mathrm{~m}^{-3}, T_{e}=50\right.$ 
eV). Direct comparison of the flux profiles (Fig. 8) shows that the deposition of plasma inside the gap can be in two different regimes. In case of small Debye length (Fig. 8A), the potential peak is well developed (maximum of potential $1.4 k T_{e}$ above the plasma potential - see Fig. 7) and the transport barrier inside the gap gives rise to a plateau region near the gap entrance (this region is not in the magnetic shadow) and then decays exponentially as described in the standard case. In case of large Debye length (Fig. 8B), there is almost no visible peak and so the decays are exponential. The transition between the 2 regimes is smooth. Dashed lines in Fig. 8 show the ballistic simulation. Similarly to the results of the standard case, there is no particle flux observed on the plasma shadowed side.

In order to quantify the plasma penetration inside the gap, we define the decay length $L_{d}$ as the distance from the gap entrance, where the flux $\Gamma_{a}$ falls bellow $1 / 1000$ of the maximum flux. In the case of small Debye length $L_{d}=0.77 \mathrm{~mm}$, while in the case of Large Debye length it doubles to $1.76 \mathrm{~mm}$. This is the main difference of the two regimes - the barrier formed in the potential dominated regime prevents ions from entering deep inside the gap.

The distribution of fluxes on gap sides shows little variation with changing plasma conditions in our parameter study. On average, $70 \%$ of the incoming flux falls on the plasma wetted side of the gap, $5 \%$ hits the plasma shadowed side and $25 \%$ is deflected and reaches the top surface of the tile. These extreme cases were compared to ballistic simulations and in both cases the fraction of deflected particles droped to $5 \%$, which verifies the role of sheath electric fields.
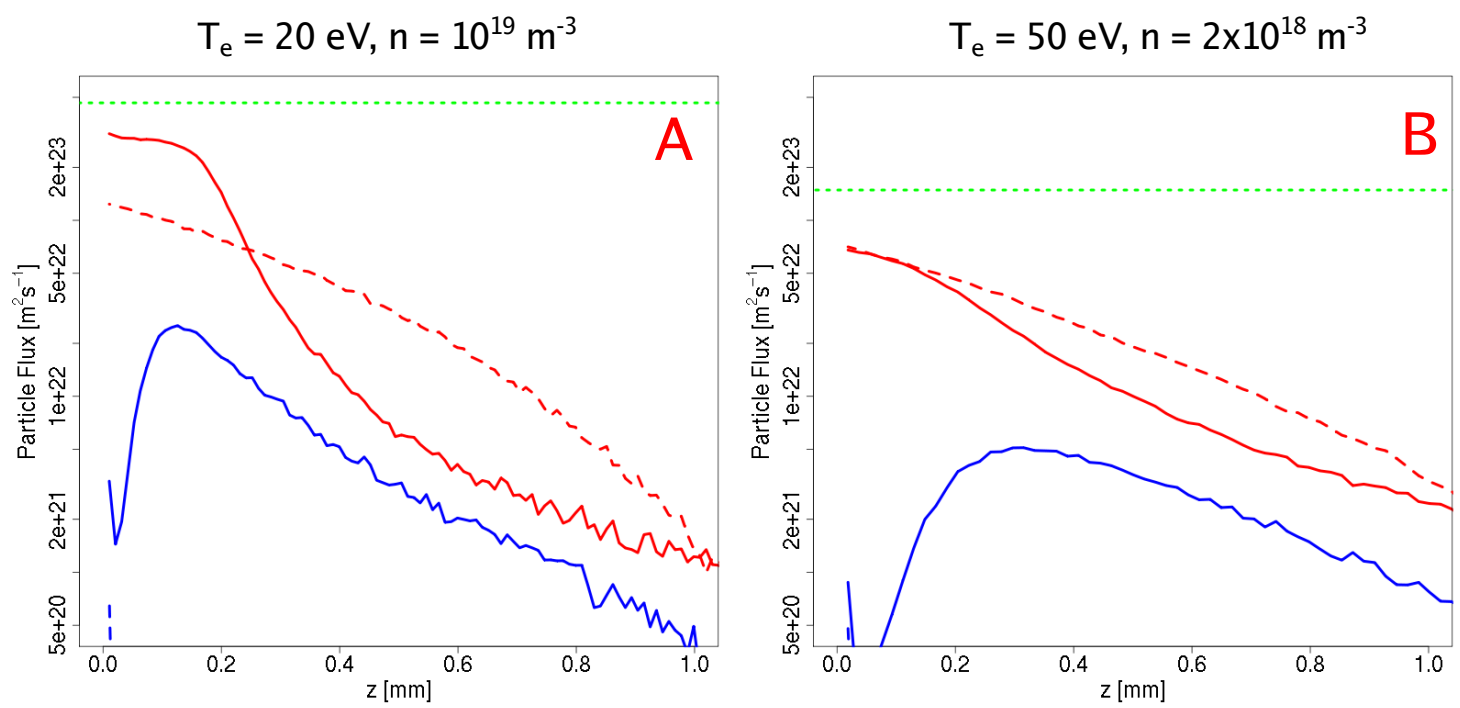

Figure 8. Deuterium ion flux profiles for the extreme cases. A - small Debye length, B - large Debye length. Plasma wetted side in red, plasma shadowed side blue. Dashed lines show the ballistic case. Green dotted lines show the projection of parallel particle flux. 


\subsection{Shaped gaps}

The purpose of tile shaping is to mitigate unfavourable transport into the gaps and protect the tile edges from extreme heat loads. Since it is unclear where to define entrance of the gap (see Fig. 9), we have decided to define the gap where the two tile sides are parallel $(z<2 \mathrm{~mm})$. The remaining part of the right tile which faces inclined top surface of the left tile ( $2 \mathrm{~mm}<z<5 \mathrm{~mm}$ ) is called Shadowed part of the right tile. A simulation for the standard conditions as used in case of an unshaped gap reveals that due to the large shadowed region, the potential peak is well developed and can rise $3 \mathrm{kT}_{e}$ above the plasma potential. This determines the transport regime in the vicinity of the gap. The flux on the right tile side is shown in Fig. 10 (right). It is seen that the flux on the shadowed part of the right tile drops quickly and there is no flux inside the gap. This behaviour has been observed for all cases with varying plasma conditions (in the same range as in case of unshaped gaps). This is an important demonstration of the effectiveness of tile shaping. The flux on the left top inclined surface (Fig. 10 left) is almost constant $(y<5 \mathrm{~mm}$ ) on the plasma wetted part of the tile and decays inside the shadowed region (separated by the black dashed line). We can note that no flux reaches the gap. Comparison with the ballistic case (blue lines) confirms that the potential peak works as a barrier for ions.

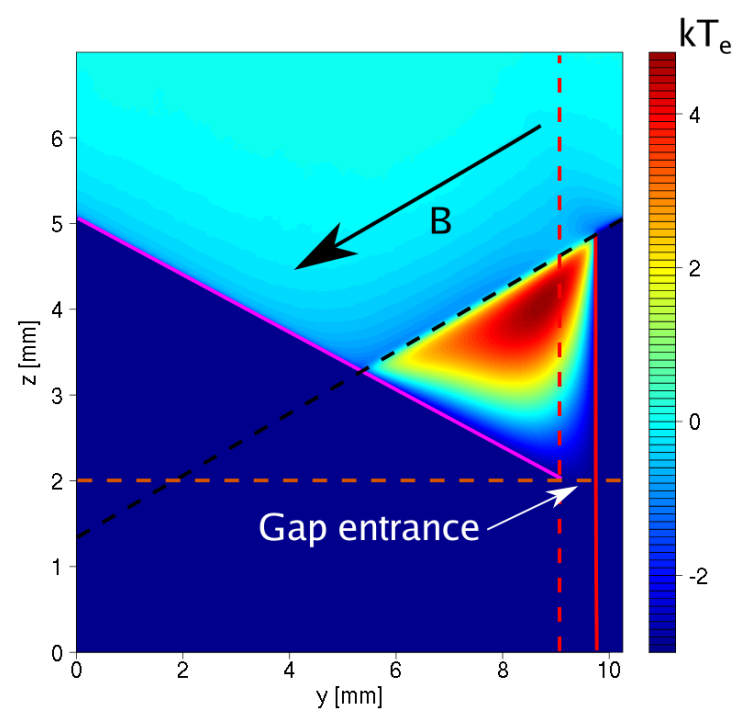

Figure 9. Potential distribution in the shaped gap for standard conditions. Magenta and red lines correspond to fluxes shown in Fig 10

In order to estimate the influence of the shaping on the plasma deposition inside the gap, the shaping parameter $H$ (see Fig. 11) was varied from $3 \mathrm{~mm}$ (strong shaping) to $0.2 \mathrm{~mm}$ (weak shaping) for the standard plasma conditions as defined in section 3.1.1. This had a direct effect on the inclination of the left tile top surface with respect to the magnetic field, which changed from 40 to 21.3 degrees. The shaping was changed so that sum of $L$ and $H$ was constant, equal to $5 \mathrm{~mm}$. The simulations show that the magnitude of the potential peak 

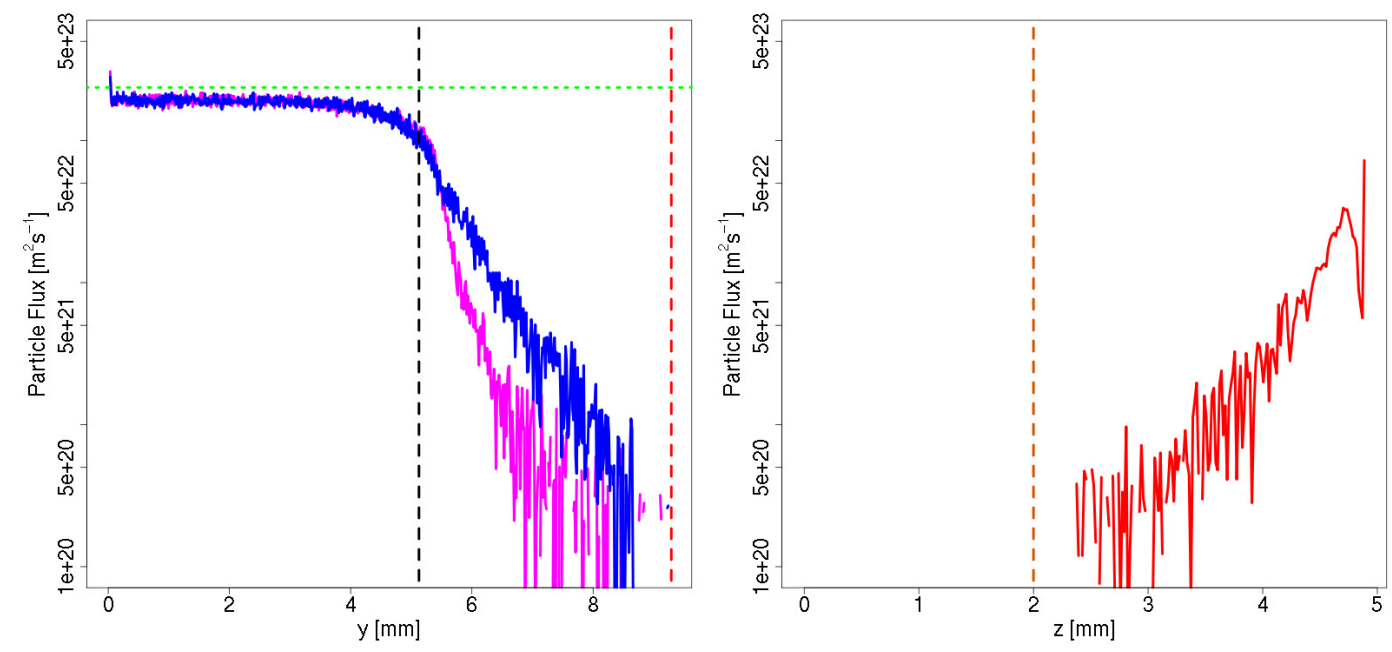

Figure 10. Particle fluxes along top left tile surface (magenta) and the right tile (red) in the shaped gap for standard conditions. The particle flux along the left tile inside the gap was negligible and as such not shown. Blue line shows the result of a ballistic case.

was decreasing with decreasing shaping but even for the weakest shaping the gap was still in potential dominated regime (potential peak maximum equals to $0.74 \mathrm{kT}_{e}$ ). The effect on the plasma fluxes is demonstrated in Fig. 12, where we have plotted the deposition profiles on both sides for varying shaping. The potential peak is effective in protecting the plasma wetted side of the gap but at the same time it pushes a fraction of the incident ions to the plasma shadowed side. For weak shaping (which is more ITER relevant), the ion flux profile on the plasma wetted side already ressembles to the unshaped case, with a typical plateau region near the gap entrance.

\section{Summary and outlook}

The plasma behaviour in the vicinity of TEXTOR tiles with shaped and unshaped gaps was simulated using the 2D3V particle-in-cell code SPICE2. The sheath electric fields near to tile surfaces are responsible for the amount of particles which enter the gap, while potential peak formed near the gap entrance was identified as the main factor influencing the plasma deposition inside the gap. Depending on the plasma conditions the ion flux profiles along the gaps are mainly influenced by the potential peak (potential dominated regime), with high flux region located near the gap entrance and fast decay for the plasma facing side of the gap, or by the geometry of the gap (geometry dominated regime) with slow exponential decay of the flux. The transition between the regimes is smooth and can be observed when the maximum of potential peak exceeds the plasma potential and so that it can effectively block ions from entering deep inside the gap. This maximum was found to be dependent on the Debye length. The main consequence of the two regimes is the influence on decay length $L_{d}$, which is twice longer $(1.76 \mathrm{~mm})$ for geometry dominated regime than for the potential dominated regime 
Strong shaping
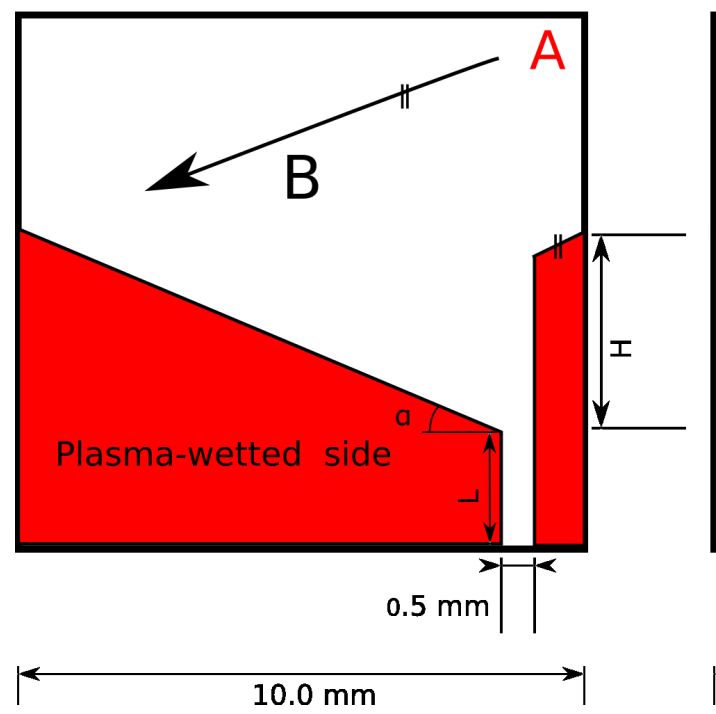

Weak shaping

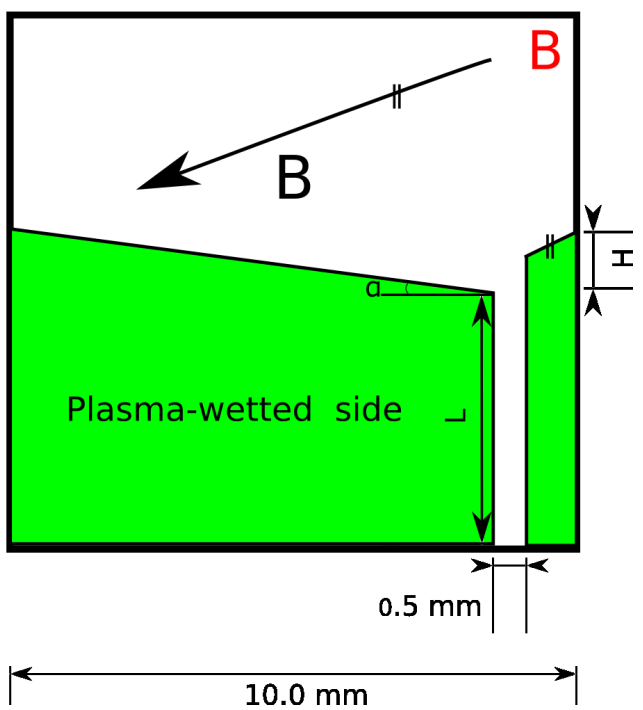

Figure 11. Geometry of the tiles with strong (left) and weak (right) shaping. The shaping parameter $H$ is varied, which affects angle $\alpha$ of the top tile surface.
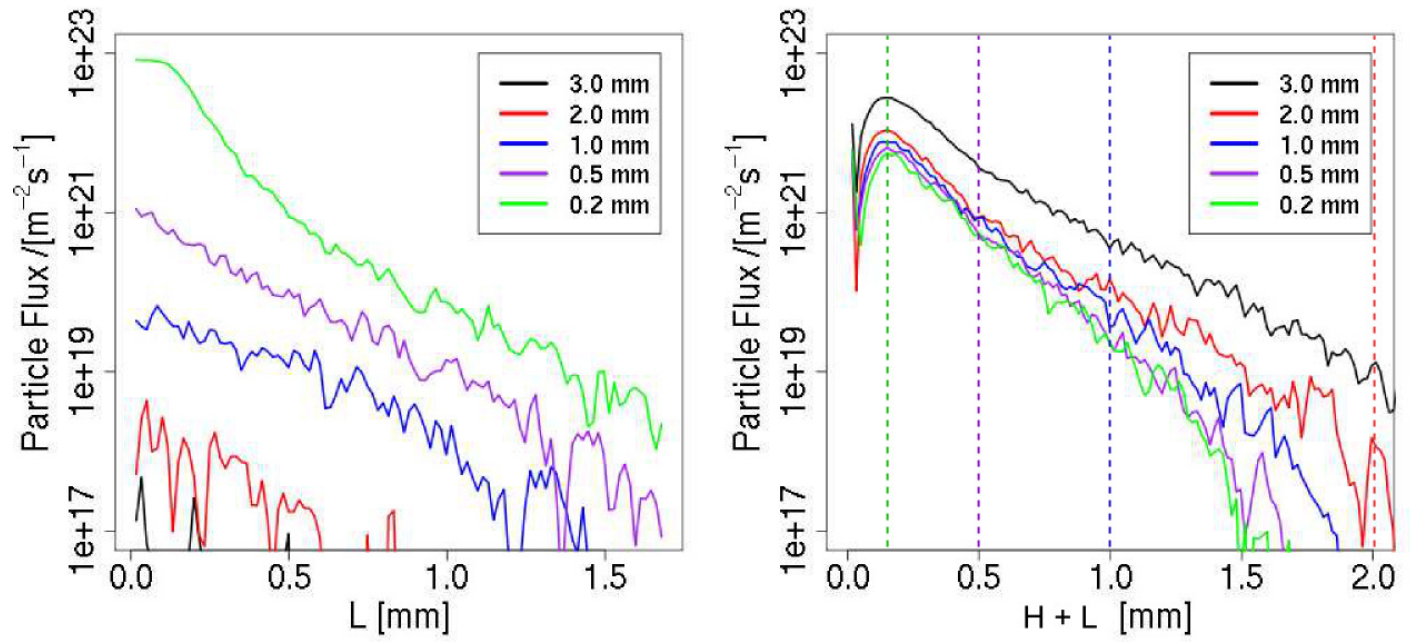

Figure 12. Deuterium flux profiles along the plasma wetted side of the gap (left) and plasma shadowed side (right) for different shaping (3.0, 2.0,1.0,0.5 and $0.2 \mathrm{~mm}$ ). Dashed lines indicate the position of the gap entrance. 
(0.77 mm) for unshaped gaps.

In case of shaped gaps, all studied cases were only in the potential dominated regime. The study of shaped gaps has shown that in case of strong shaping there is a large potential peak which can protect the gap from incoming ion flux, however such geometry would result in elevated heat fluxes on the top tile surface (the magnetic field has inclination of 40 degrees with respect to the top surface of the tile). There is also a significant ion flux deflected by the potential peak onto the plasma shadowed side of the gap. In case of weak shaping the flux profiles indicate a transition to the unshaped gap. The fluxes obtained from the simulations were used as input for the 3D-GAPS code [5] to study hydrocarbon layer formation inside the gaps.

Future studies will involve simulations of more ITER relevant geometries with magnetic field at oblique angle with respect to the top tile surface. Such simulations should verify the applicability of tile shaping for the ITER divertor. The simulations will also include carbon (as done in [11]) in order to study its penetration into the shaped gaps. Since ITER divertor plasma will be of high density $\left(10^{20}-10^{21} \mathrm{~m}^{-3}\right)$ and low temperature $(1-2 \mathrm{eV})$, the Debye length will be substantially reduced comparing to cases studied in this work. This will make the simulations much more demanding and will probably require further development of the SPICE2 code to allow parallelization on higher number of processors.

\section{Acknowledgments}

This work was supported by Euratom. The views and opinions expressed herein do not necessarily reflect those of the European Commission. The work was supported by the grant GA CR No. 202/09/1467. Simulations for this work were performed at computational cluster LUNA at Institute of Physics of the ASCR, v.v.i.

[1] T. Loarer, J. of Nucl. Mater., 390-391, 20-28, 2009.

[2] D.S. Walsh, B.L. Doyle, G.L. Jackson, J. Vac. Sci. Technol. A, 10, 1174-1179, 1992.

[3] A. Litnovsky et al.,Phys. Scr., T128, 2007.

[4] A. Litnovsky et al.,J. of Nucl. Mater., 390-391, 2009.

[5] D. Matveev, A. Kirschner, A. Litnovsky, M. Komm, D. Borodin, V. Philipps, G. van Oost, Plasma Phys. Control. Fusion 52 (2010) 075007

[6] C. K. Birdsall, and A. B. Langdon, McGraw-Hill, 1985, 1991

[7] R. Dejarnac and J.P. Gunn, J. Nucl. Mater., 363-365, 560-564, 2007.

[8] J.P. Gunn, J. Nucl. Mater. 337-339, 310, 2005.

[9] R. Dejarnac, M. Komm, J. Stockel and R. Panek J. Nucl. Mater., 382, 31-34, 2008.

[10] R. Dejarnac, M. Komm, J.P. Gunn and R. Panek, J. Nucl. Mater., 390-391, 818-821, 2009.

[11] R. Dejarnac, M. Komm, D. Tskhakaya, J. P. Gunn, Z. Pekarek, IEEE Trans. on Plasma Phys.,38, 10421046, 2010. 